\title{
Alpha-stat, beach chair, cerebral autoregulation... But who is at risk?
}

\author{
John M. Murkin, MD • Mauricio Giraldo, MD
}

Received: 2 September 2015/Revised: 14 October 2015/Accepted: 27 January 2016/Published online: 2 March 2016

(c) Canadian Anesthesiologists' Society 2016

At a recent Institutional Research Ethics Board meeting, an experienced intensivist commented, "The more we learn about cerebral autoregulation (CA) the less we know...", upon learning of a current transcranial Doppler (TCD) study that was unable to show a cerebral autoregulatory plateau in healthy volunteers. ${ }^{1}$

Our experience is that most clinicians have much less understanding of CA than is generally considered. Indeed, rather than occurring as a relatively static phenomenon showing a zero-slope autoregulatory plateau from 50-150 $\mathrm{mmHg}$ mean arterial pressure (MAP), vector analysis instead reflects a series of cascading parallelograms of variable slope determined by the regional cerebral metabolic rate (CMR). ${ }^{2}$ Furthermore, $\mathrm{CA}$ is also reflected by the tight and fundamental link between regional cerebral blood flow (CBF) and CMR for which $\mathrm{CO}_{2}$ is a primary driver over a range governed by regional vasoreactivity. The upper and lower slopes represent the limits of cerebral vasoconstriction and vasodilation, whether or not these limits are related to primary vasomotor effects or include other driving factors, such as regional viscosity, ${ }^{3}$ wall stress, atherosclerotic burden, and other related phenomena, including alterations to endothelial glycocalyx ${ }^{4}$ such as may occur with microgaseous emboli (MGE). ${ }^{5}$

J. M. Murkin, MD ( $₫)$

Department of Anesthesiology and Perioperative Medicine, University of Western Ontario, Rm C3-112 University Hospital, 339 Windermere Rd, London, ON N6A 5A5, Canada e-mail: john.murkin@lhsc.on.ca

M. Giraldo, MD

Department of Anesthesia, London Health Sciences Centre, London, ON, Canada
This was illustrated most dramatically by Kollmar et al. in a study of CBF during alpha-stat vs pH-stat ventilatory management in eight patients with ischemic brain injury treated with mild hypothermia. ${ }^{6}$ The resulting $7-8 \mathrm{mmHg}$ increase in $\mathrm{PaCO}_{2}$ content consequent with $\mathrm{pH}$-stat utilization produced substantial increases in $\mathrm{CBF}$ and resulted in brainstem herniation requiring resuscitation in two of the eight patients. Importantly, this rather modest manipulation of $\mathrm{CO}_{2}$ would be commonly seen with the type of ventilatory manipulations that occur routinely in clinical practice. The important difference between routine intraoperative patient management and the eight study patients - apart from having their CBF actively studied - was their high degree of susceptibility to small changes in $\mathrm{CBF}$ due to ongoing ischemia. What would be considered otherwise subtle $\mathrm{CO}_{2}$ changes could produce catastrophic consequences in susceptible patients. But who else is at risk?

Aaslid et al. provided a fundamental insight into CA by using TCD to focus on the dynamic phase of CA pressure/vasoreactivity. ${ }^{7}$ They identified a $0.2-0.4 \mathrm{~Hz}$ (2-5 $\mathrm{sec})$ response time characterized by transfer function analysis (TFA) in terms of amplitude, phase shift, and latency. This ultradynamic flow/metabolism linkage has subsequently been used to characterize normal and abnormal states of dynamic CA (dCA) autoregulatory integrity. While some of the particulars of dCA TFA remain contentious, ${ }^{8}$ investigations employing time domain analysis and utilizing a moving linear correlation coefficient between incremental changes in MAP vs changes in various CBF-related parameters (e.g., intracranial pressure, TCD, or near-infrared spectroscopy $)^{9-11}$ enable detection of the presence or absence of dCA and the ability to detect the lower limit of cerebral autoregulation (LLA) using correlation analysis. $^{12}$ One of the clinical implications of $\mathrm{dCA}$ 
analysis is the ability to derive the presence or absence of CA and the LLA from spontaneous changes in MAP, both continuously and without provoking a step change in perfusion pressure. This has been successfully employed in head-injured patients and more recently in perioperative cardiac surgical patients. ${ }^{13-16}$

The data from cardiac surgical patients is particularly germane and shows a surprisingly wide range of LLA during cardiopulmonary bypass (CPB) - absent in some patients and significantly greater than $50 \mathrm{mmHg}$ in others. ${ }^{14,15}$ Investigations in these CPB patients have also reported an increased incidence of postoperative stroke as well as other end-organ damage in patients in whom MAP was below LLA. ${ }^{14,16,17}$ These studies and others in preoperative patients ${ }^{18}$ have underscored the validity of the concept of the "brain as the index organ", as initially described in a widely disseminated clinical study on the impact of cerebral oximetry monitoring ${ }^{19}$ and subsequently confirmed in a more recent series of separate outcomes studies. ${ }^{17,20,21}$ Our own data ${ }^{15}$ have shown the surprising variability of the LLA and have further underscored the impact that CPB-related changes (e.g., laminar flow, viscosity, etc.) have on the integrity of CA. Again, who is susceptible?

And if the brain is being hypoperfused - the brain being the organ most conspicuous for autoregulation - how is perfusion to the gut and kidneys and other organs that may have an even greater burden of pre-morbid impairment? Indeed, the concept of the "brain as the index organ" does not imply simply that if the brain is $O K$ - everything is $O K$, but rather, it clearly indicates that if the brain is "not OK"- then be aware, and beware. ${ }^{22}$ Below the LLA, end-organ viability becomes uncertain and is increasingly dependent upon a variety of interactions as influenced particularly by comorbid conditions, including drugs (e.g., statins) and the state of arousal and magnitude of the inflammatory response. ${ }^{23,24}$

One of the implications of our early study of $\mathrm{PaCO}_{2}$ on $\mathrm{CBF}$ and $\mathrm{CMR}$ during hypothermic $\mathrm{CPB}^{25}$ - aside from overturning routine use of $\mathrm{pH}$-stat, one of the then fundamentals of clinical management ${ }^{26}$ - was the recognition that inappropriate increases in $\mathrm{PaCO}_{2}$ can disproportionately increase $\mathrm{CBF}$. Thus follows the potential for increased delivery of gaseous and particulate microemboli into the cerebral circulation, ${ }^{25}$ with a consequent increase in adverse neurobehavioural outcomes. ${ }^{27,28}$ Nevertheless, until recently, the exponentially greater cerebral delivery of MGE during open-chamber valve surgery compared with closedchamber coronary artery bypass $(\mathrm{CAB})$ paradoxically did not appear to be strongly causally related to adverse clinical outcomes. ${ }^{29}$
Nevertheless, this potential impact of increased MGE and adverse events changed with the expanded use of tranexamic acid (TXA) following the withdrawal of aprotinin from clinical use. When our group, in concert with colleagues at Hammersmith Hospital in London, UK, first identified the occurrence of non-ischemic postoperative seizures in patients who received highdose $\mathrm{TXA}^{30}$ one of the striking findings was the preponderance of seizures in patients who underwent open-chamber procedures $v s$ coronary bypass surgery. This observation was subsequently confirmed in numerous clinical reports of TXA toxicity that have followed. ${ }^{31,32}$ It is thus hard to refute the contention that increased blood brain barrier permeability secondary to increased cerebral MGE elevates cerebrospinal fluid TXA levels into toxic concentrations $^{33}$ - MGE from open-chamber surgery having rendered these patients particularly susceptible to TXA toxicity.

So who is at risk? There have been various debates over cerebral perfusion while in the beach chair position (BCP). ${ }^{34}$ On the one hand, some regard it as a "waterfall" in which the difference between the heart (right atrium) and the brain (auditory meatus) should be considered (i.e., for each centimetre of vertical height, there is a reduction of $0.77 \mathrm{mmHg}$ in cerebral perfusion pressure). Others, however, regard it as a "siphon" for which the measurement of this difference and the place where it is measured (e.g., arm, calf) are not important (i.e., the effects of gravity are the same for the upward arterial blood column as for blood returning to the heart). Whether it is as considered in the above two schemes, or if even more complex physiology at work, it is clear that some threshold MAP is required for cerebral perfusion.

And so, in this issue of the Journal, Meex et al. provide another benchmark for comparing the incidence of significant (i.e., $>20 \%$ ) position-related cerebral desaturation. ${ }^{35}$ The authors studied 195 anesthetized patients; 101 patients were placed in the BCP and 94 were placed in the lateral decubitus position (LDP). Study results showed a $57 \%$ incidence of significant cerebral desaturation in the BCP group vs $6 \%$ in the LDP group. None of 85 non-anesthetized healthy volunteers showed significant cerebral desaturation in either body position. While clinical outcomes are not reported in the current study, however, it is likely that the degree of cerebral desaturation seen in these patients would not have been associated with any detectable cerebral injury. This was shown most recently by Laflam et al., in a study comparably sized to the current one, which showed no differences in cognitive outcomes or brain injury biomarkers between the BCP patients and the LDP patients despite showing impaired cerebral autoregulation 
and significantly lower cerebral saturation associated with BCP. $^{36}$

Nevertheless, there clearly remains an extremely rare but devastatingly serious incidence of neurological injury reported for BCP patients. Unlike cardiac surgery, however, where cerebral desaturation and impairment of CA - likely operating in concert with MGE, dilutional anemia, an exaggerated inflammatory response, and a variety of other factors - have been linked repeatedly to major adverse outcomes, significant (i.e., > 20\%) cerebral desaturation alone does not appear sufficient to cause clinically detectable injury in the majority of BCP patients. Not sufficient by itself, but significant (i.e., $>20 \%$ ) cerebral desaturation in BCP is likely a necessary condition that further increases susceptibility to injury yet becomes manifest as overt cerebral injury only when some further condition is present.

As outlined above, ever more focused patient monitoring and the attendent individualization of clinical care are fundamental steps towards further enhancing patient safety. Again, who is at risk?

\section{Alpha-stat, position semi-assise, autorégulation cérébrale... Mais qui est à risque?}

Lors de la dernière réunion du Comité d'éthique de la recherche de notre institution, un intensiviste d'expérience a commenté : «Plus nous apprenons de choses sur l'autorégulation cérébrale (AC), moins nous en savons... », en apprenant qu'une étude en cours sur le Doppler transcrânien (DTC) n'avait pas pu déterminer de plateau d'autorégulation cérébrale chez des volontaires sains. ${ }^{1}$

En nous fondant sur notre expérience, nous pouvons avancer que les cliniciens comprennent bien moins l'AC que ce qui est généralement présumé. En effet, au lieu d'être un phénomène relativement statique montrant un plateau d'autorégulation à pente zéro entre 50 et 150 $\mathrm{mmHg}$ de tension artérielle moyenne (TAM), l'analyse vectorielle de l'AC reflète en fait une série de parallélogrammes en cascade de pentes variables, lesquelles sont déterminées par le taux métabolique cérébral (TMC) régional. $^{2}$ De plus, l'AC se reflète également par le lien serré et fondamental entre la circulation sanguine cérébrale (CSC) régionale et le TMC, pour lequel le $\mathrm{CO}_{2}$ constitue le principal moteur sur un éventail régi par la vasoréactivité régionale. Les pentes supérieures et inférieures représentent les limites de la vasoconstriction et de la vasodilatation cérébrale, que ces limites soient ou non liées à des effets vasomoteurs primaires ou qu'elles comportent d'autres facteurs déclencheurs, tels que la viscosité régionale, ${ }^{3}$ la tension pariétale, le fardeau d'athérosclérose et d'autres phénomènes liés, y compris des modifications de la glycocalyx endothéliale ${ }^{4}$ telles que celles survenant en cas de micro-embolies gazeuses (MEG). ${ }^{5}$

Ce phénomène a été illustré de façon spectaculaire par Kollmar et coll. dans une étude de la CSC pendant la prise en charge ventilatoire en alpha-stat $v s$ en pH-stat de huit patients souffrant d'une lésion cérébrale ischémique et traités à l'aide d'une légère hypothermie. ${ }^{6}$ L'augmentation résultante de 7-8 mmHg du contenu de $\mathrm{PaCO}_{2}$, qui coïncidait avec l'utilisation de $\mathrm{pH}$-stat, a provoqué des augmentations considérables de la CSC et entraîné un engagement du tronc cérébral nécessitant la réanimation de deux des huit patients. Il est important de souligner que cette manipulation plutôt modeste du $\mathrm{CO}_{2}$ serait courante avec le type de manipulations ventilatoires qui surviennent régulièrement dans la pratique clinique. La différence importante entre la prise en charge peropératoire de routine d'un patient et les huit patients de l'étude - hormis l'étude active de leur CSC - était leur degré élevé de susceptibilité à de légères modifications de la CSC en raison d'une ischémie continue. Ce qui serait autrement considéré comme des changements subtils de $\mathrm{CO}_{2}$ pourrait avoir des conséquences catastrophiques chez des patients susceptibles. Mais qui d'autre est à risque?

Dans leur étude, Aaslid et coll. proposent une perspective fondamentale de l'AC en se servant du DTC pour se concentrer sur la phase dynamique de pression / vasoréactivité de $1^{\prime} \mathrm{AC}{ }^{7}$ Ils ont identifié un temps de réponse de $0,2-0,4 \mathrm{~Hz}(2-5 \mathrm{sec})$ caractérisé par une analyse de fonction de transfert (AFT) en termes d'amplitude, de déphasage et de latence. Cette liaison ultradynamique entre le débit et le métabolisme a par la suite été utilisée pour caractériser les états normaux et anormaux d'intégrité d'autorégulation d'une $\mathrm{AC}$ dynamique (ACd). Bien que certaines des particularités de l'AFT d'une ACd demeurent controversées, ${ }^{8}$ des recherches se fondant sur l'analyse du domaine temps et utilisant un coefficient de corrélation linéaire mobile entre les changements incrémentiels de la TAM par rapport aux changements observés dans divers paramètres liés à la CSC (par ex. la pression intracrânienne, le DTC, ou la spectroscopie proche infrarouge $)^{9-11}$ permettent de détecter la présence ou l'absence d'une $\mathrm{ACd}$ et de déterminer la limite inférieure de l'autorégulation cérébrale (LIAC) à l'aide d'une analyse de corrélation. $^{12}$ L'une des implications cliniques de l'analyse de l'ACd est la capacité de dériver la présence ou l'absence d'AC et la LIAC à partir de changements spontanés de la TAM, à la fois en continu et sans provoquer 
de changement d'étape dans la pression de perfusion. Cette méthode a été utilisée avec succès chez des patients présentant des lésions à la tête et, plus récemment, chez des patients de chirurgie cardiaque en période périopératoire. $^{13-16}$

Les données recueillies auprès des patients de chirurgie cardiaque sont particulièrement pertinentes et montrent un éventail étonnamment étendu de LIAC pendant la circulation extracorporelle (CEC) - d'absente chez certains patients à considérablement plus élevée que 50 mmHg chez d'autres. ${ }^{14,15}$ Les recherches menées auprès de ces patients avec CEC ont également révélé une incidence accrue d'accident vasculaire cérébral (AVC) postopératoire ainsi que d'autres atteintes des organes cibles chez les patients dont la TAM était inférieure à la LIAC. ${ }^{14,16,17} \mathrm{Ces}$ études et d'autres réalisées auprès de patients en période préopératoire $^{18}$ ont souligné la validité du concept de « cerveau en tant qu'organe de référence », comme cela avait été originalement décrit dans une étude clinique largement diffusée sur l'impact du monitorage de l'oxymétrie cérébrale, ${ }^{19}$ puis confirmé par la suite dans une série plus récente d'études de résultats séparées. ${ }^{17,20,21}$ Nos propres données ont démontré la variabilité surprenante de la LIAC et soulignent davantage encore l'impact que des changements liés à la CEC (par ex. flux laminaire, viscosité, etc.) ont sur l'intégrité de l'AC. La question se pose à nouveau : qui est susceptible?

Et si le cerveau est hypoperfusé - le cerveau étant l'organe le plus évident en matière d'autorégulation - dans quel état se trouve la perfusion de l'intestin, des reins et d'autres organes qui pourraient être encore plus affectés par une insuffisance prémorbide? En effet, le concept du «cerveau comme organe de référence» ne veut pas simplement dire que si le cerveau va bien, tout va bien. Plutôt, il indique clairement que si le cerveau ne va pas bien, alors ouvre les yeux et prends garde. ${ }^{22}$ En-dessous de la LIAC, la viabilité des organes cibles devient incertaine et dépend de plus en plus de diverses interactions telles qu'elles sont influencées tout particulièrement par des conditions comorbides, notamment les médicaments (par ex. les statines) et l'état d'excitation et la magnitude de la réponse inflammatoire. . $3,24^{24}$

L'une des implications de notre première étude de la $\mathrm{PaCO}_{2}$ sur la CSC et le TMC pendant une CEC en hypothermie $^{25}$ - outre le fait de renverser l'utilisation de routine de la $\mathrm{pH}$-stat - l'un des fondements de la prise en charge clinique de l'époque ${ }^{26}$ - était la prise de conscience que des augmentations inadaptées de la $\mathrm{PaCO}_{2}$ pouvaient augmenter de façon disproportionnée la CSC. Ainsi s'ensuit le potentiel d'une diffusion accrue de micro-embolies gazeuses et particulaires dans la circulation cérébrale, ${ }^{25}$ avec une augmentation subséquente des complications neurocomportementales. ${ }^{27,28}$ Toutefois, jusqu'à récemment, la diffusion cérébrale exponentiellement accrue de MEG pendant la chirurgie valvulaire à cœur ouvert par rapport à un pontage aortocoronarien (PAC) n'a pas, paradoxalement, semblé avoir une relation causale forte avec des complications cliniques. ${ }^{29}$

Néanmoins, cet impact potentiel de MEG accrues et de complications a changé avec l'utilisation plus répandue de l'acide tranexamique (TXA) après le retrait de l'aprotinine de l'usage clinique. Lorsque notre groupe, de concert avec nos collègues de l'Hôpital de Hammersmith de Londres, au Royaume-Uni, a remarqué pour la première fois la survenue de crises postopératoires non ischémiques chez les patients ayant reçu du TXA à forte dose ${ }^{30}$ l'une des découvertes étonnantes était la prépondérance de crises chez les patients subissant des interventions à cœur ouvert par rapport à ceux subissant des chirurgies de pontage. Cette observation a été confirmée par la suite dans plusieurs comptes rendus cliniques de toxicité du TXA. ${ }^{31,32}$ Dès lors, il est difficile de réfuter l'affirmation qu'une perméabilité accrue de la barrière hémato-encéphalique secondaire à des MEG cérébrales accrues élève les niveaux de TXA dans le liquide céphalorachidien à des concentrations toxiques $^{33}$ - les MEG de la chirurgie à cœur ouvert ayant rendu ces patients particulièrement susceptibles à une toxicité du TXA.

Donc, qui est à risque? Plusieurs débats ont porté sur la perfusion cérébrale en position semi-assise. ${ }^{34}$ D'une part, certains considèrent cette position comme une «chute d'eau » dans laquelle la différence entre le cœur (oreillette droite) et le cerveau (méat auditif externe) devrait être examinée (c.-à-d. pour chaque centimètre de hauteur verticale, il y a une réduction de $0,77 \mathrm{mmHg}$ de la pression de perfusion cérébrale). D'autres, toutefois, la considèrent comme un "siphon ", dans quel cas la mesure de cette différence et l'emplacement de la mesure (par ex. le bras, le mollet) n'ont aucune importance (c.à-d. les effets de la gravité sont les mêmes pour la colonne de sang artériel ascendante que pour le sang retournant au cœur). Qu'elle soit envisagée selon l'un de deux schémas décrits ci-dessus ou en tant qu'une physiologie encore plus complexe, il est clair qu'une TAM minimale est requise pour la perfusion cérébrale.

Et c'est pourquoi, dans ce numéro du Journal, Meex et coll. proposent un autre jalon pour comparer l'incidence de désaturation cérébrale significative (soit $>20 \%$ ). ${ }^{35}$ Les auteurs ont étudié 195 patients anesthésiés; 101 patients ont été placés en position semi-assise et 94 en décubitus latéral. Les résultats de l'étude ont montré une incidence de $57 \%$ de désaturation cérébrale significative dans le groupe semi-assis vs $6 \%$ dans le groupe décubitus latéral. Aucun des 85 volontaires sains non anesthésiés n'a montré de désaturation cérébrale significative. Bien que les résultats cliniques ne soient pas rapportés dans cette 
étude, il est probable que le degré de désaturation cérébrale observé chez ces patients n'aurait pas été associé à une quelconque lésion cérébrale dépistable. Laflam et coll. l'ont récemment démontré dans une étude d'envergure similaire à celle présentée ici, où aucune différence en matière de pronostics cognitifs ou de biomarqueurs de lésion cérébrale entre les patients en position semi-assise et ceux en décubitus latéral n'a été observée, malgré la présence d'une autorégulation cérébrale entravée et d'une saturation cérébrale significativement plus basse associées à la position semi-assise. ${ }^{36}$

Il demeure cependant clairement une incidence, certes extrêmement rare, mais gravissime, de lésion neurologique rapportée chez les patients en position semi-assise. Au contraire de la chirurgie cardiaque, dans laquelle la désaturation cérébrale et la dégradation de l'AC - fonctionnant probablement de concert avec les MEG, l'anémie dilutionnelle, une réponse inflammatoire exagérée et divers autres facteurs - a été associée, à plusieurs reprises, à des complications majeures, une désaturation cérébrale significative (soit $>20 \%$ ) seule ne semble pas suffire à provoquer une lésion dépistable d'un point de vue clinique chez la majorité des patients en position semi-assise. Une importante désaturation cérébrale (soit $>20 \%$ ) en position semi-assise, bien qu'insuffisante en soi, constitue probablement une condition nécessaire, qui augmente encore davantage la susceptibilité aux lésions, mais qui ne se manifeste sous forme de lésion cérébrale flagrante que lorsqu'une autre condition est présente. À nouveau, qui est à risque?

Conflicts of interest None declared.

Conflit d'intérêt Aucun.

\section{References}

1. Lucas SJ, Tzeng YC, Galvin SD, Thomas KN, Ogoh S, Ainslie PN. Influence of changes in blood pressure on cerebral perfusion and oxygenation. Hypertension 2010; 55: 698-705.

2. Murkin JM. Cerebral autoregulation: the role of $\mathrm{CO} 2$ in metabolic homeostasis. Semin Cardiothorac Vasc Anesth 2007; 11: 269-73.

3. Bia D, Aguirre I, Zocalo Y, Devera L, Cabrera Fischer E, Armentano $R$. Regional differences in viscosity, elasticity and wall buffering function in systemic arteries: pulse wave analysis of the arterial pressure-diameter relationship (Spanish). Rev Esp Cardiol 2005; 58: 167-74.

4. Vogel J, Sperandio $M$, Pries AR, Linderkamp O, Gaehtgens $P$, Kuschinsky $W$. Influence of the endothelial glycocalyx on cerebral blood flow in mice. J Cereb Blood Flow Metab 2000; 20: $1571-8$.

5. Eckmann DM, Armstead SC. Influence of endothelial glycocalyx degradation and surfactants on air embolism adhesion. Anesthesiology 2006; 105: 1220-7.
6. Kollmar R, Georgiadis D, Schwab S. Alpha-stat versus pH-stat guided ventilation in patients with large ischemic stroke treated by hypothermia. Neurocrit Care 2009; 10: 173-80.

7. Aaslid $R$, Lindegaard KF, Sorteberg W, Nornes H. Cerebral autoregulation dynamics in humans. Stroke 1989; 20: 45-52.

8. Meel-van den Abeelen AS, Simpson DM, Wang LJ, et al. Between-centre variability in transfer function analysis, a widely used method for linear quantification of the dynamic pressure-flow relation: the CARNet study. Med Eng Phys 2014; 36: 620-7.

9. Steiner LA, Coles JP, Czosnyka M, et al. Cerebrovascular pressure reactivity is related to global cerebral oxygen metabolism after head injury. J Neurol Neurosurg Psychiatry 2003; 74: 765-70.

10. Zweifel C, Czosnyka M, Lavinio A, et al. A comparison study of cerebral autoregulation assessed with transcranial Doppler and cortical laser Doppler flowmetry. Neurol Res 2010; 32: 425-8.

11. Ono M, Zheng Y, Joshi B, Sigl JC, Hogue CW. Validation of a stand-alone near-infrared spectroscopy system for monitoring cerebral autoregulation during cardiac surgery. Anesth Analg 2013; 116: 198-204.

12. Brady KM, Lee JK, Kibler KK, et al. Continuous time-domain analysis of cerebrovascular autoregulation using near-infrared spectroscopy. Stroke 2007; 38: 2818.

13. Zweifel C, Dias $C$, Smielewski $P, C$ zosnyka $M$. Continuous timedomain monitoring of cerebral autoregulation in neurocritical care. Med Eng Phys 2014; 36: 638-45.

14. Ono M, Joshi B, Brady $K$, et al. Risks for impaired cerebral autoregulation during cardiopulmonary bypass and postoperative stroke. Br J Anaesth 2012; 109: 391-8.

15. Murkin JM, Kamar M, Silman Z, Balberg M, Adams SJ. Intraoperative cerebral autoregulation assessment using ultrasound-tagged near-infrared-based cerebral blood flow in comparison to transcranial Doppler cerebral flow velocity: a pilot study. J Cardiothorac Vasc Anesth 2015; 29: 1187-93.

16. Ono M, Arnaoutakis GJ, Fine DM, et al. Blood pressure excursions below the cerebral autoregulation threshold during cardiac surgery are associated with acute kidney injury. Crit Care Med 2013; 41: 464-71.

17. Ono M, Brady K, Easley RB, et al. Duration and magnitude of blood pressure below cerebral autoregulation threshold during cardiopulmonary bypass is associated with major morbidity and operative mortality. J Thorac Cardiovasc Surg 2014; 147: 483-9.

18. Heringlake $M$, Garbers $C$, Kabler JH, et al. Preoperative cerebral oxygen saturation and clinical outcomes in cardiac surgery. Anesthesiology 2011; 114: 58-69.

19. Murkin JM, Adams SJ, Novick RJ, et al. Monitoring brain oxygen saturation during coronary bypass surgery: a randomized, prospective study. Anesth Analg 2007; 104: 51-8.

20. Fischer GW, Lin HM, Krol M, et al. Noninvasive cerebral oxygenation may predict outcome in patients undergoing aortic arch surgery. J Thorac Cardiovasc Surg 2011; 141: 815-21.

21. Kazan R, Bracco D, Hemmerling TM. Reduced cerebral oxygen saturation measured by absolute cerebral oximetry during thoracic surgery correlates with postoperative complications. $\mathrm{Br}$ J Anaesth 2009; 103: 811-6.

22. Murkin JM. Cerebral oximetry: monitoring the brain as the index organ. Anesthesiology 2011; 114: 12-3.

23. Murkin JM. Panvascular inflammation and mechanisms of injury in perioperative CNS outcomes. Semin Cardiothorac Vasc Anesth 2010; 14: 190-5.

24. Grau AJ, Boddy AW, Dukovic DA, et al. CAPRIE Investigators. Leukocyte count as an independent predictor of recurrent ischemic events. Stroke 2004; 35: 1147-52.

25. Murkin JM, Farrar JK, Tweed WA, McKenzie FN, Guiraudon G. Cerebral autoregulation and flow/metabolism coupling during 
cardiopulmonary bypass: the influence of $\mathrm{PaCO} 2$. Anesth Analg 1987; 66: 825-32.

26. Hindman BJ. Choice of alpha-stat or $\mathrm{pH}$-stat management and neurologic outcomes after cardiac surgery: it depends. Anesthesiology 1998; 89: 5-7.

27. Murkin JM, Martzke JS, Buchan AM, Bentley C, Wong CJ. A randomized study of the influence of perfusion technique and $\mathrm{pH}$ management strategy in 316 patients undergoing coronary artery bypass surgery. II. Neurologic and cognitive outcomes. J Thorac Cardiovasc Surg 1995; 110: 349-62.

28. Patel RL, Turtle MR, Chambers DJ, James DN, Newman S, Venn $G E$. Alpha-stat acid-base regulation during cardiopulmonary bypass improves neuropsychologic outcome in patients undergoing coronary artery bypass grafting. J Thorac Cardiovasc Surg 1996; 111: 1267-79.

29. Neville MJ, Butterworth J, James RL, Hammon JW, Stump DA. Similar neurobehavioral outcome after valve or coronary artery operations despite differing carotid embolic counts. J Thorac Cardiovasc Surg 2001; 121: 125-36.

30. Murkin JM, Falter F, Granton J, Young B, Burt C, Chu M. High-dose tranexamic acid is associated with nonischemic clinical seizures in cardiac surgical patients. Anesth Analg 2010; 110: 350-3.

31. Koster A, Borgermann J, Zittermann A, Lueth JU, GillisJanuszewski T, Schirmer U. Moderate dosage of tranexamic acid during cardiac surgery with cardiopulmonary bypass and convulsive seizures: incidence and clinical outcome. Br J Anaesth 2013; 110: 34-40.

32. Manji RA, Grocott HP, Leake J, et al. Seizures following cardiac surgery: the impact of tranexamic acid and other risk factors. Can J Anesth 2012; 59: 6-13.

33. Lecker I, Wang DS, Romaschin AD, Peterson M, Mazer CD, Orser BA. Tranexamic acid concentrations associated with human seizures inhibit glycine receptors. J Clin Invest 2012; 122: 4654-66.

34. Drummond JC. A beach chair, comfortably positioned atop an iceberg. Anesth Analg 2013; 116: 1204-6.

35. Meex I, Vundelinckx J, Buyse K, et al. Cerebral tissue oxygen saturation values in volunteers and patients in the lateral decubitus and beach chair positions: a prospective observational study. Can J Anesth 2016; 63: this issue. DOI: 10.1007/s12630016-0604-3

36. Laflam A, Joshi B, Brady K, et al. Shoulder surgery in the beach chair position is associated with diminished cerebral autoregulation but no differences in postoperative cognition or brain injury biomarker levels compared with supine positioning: the anesthesia patient safety foundation beach chair study. Anesth Analg 2015; 120: 176-85. 